\title{
Introspective Interest and Insight in the Context of Mindfulness-Based Stress Reduction: a Randomized Trial
}

\author{
Ivan Nyklíček ${ }^{1}$ (D) $\cdot$ Renée Zonneveld ${ }^{1} \cdot$ Johan Denollet $^{1}$
}

Published online: 11 July 2020

(C) The Author(s) 2020

\begin{abstract}
Objective Introspective interest and insight have long been argued to be important factors in psychotherapy. However, empirical studies are scarce and their results equivocal. Therefore, in the present study, the potential moderating and mediating roles of introspective interest and insight were examined in the context of mindfulness-based stress reduction (MBSR).

Methods In a randomized controlled trial, 72 MBSR participants were compared with 71 waitlist control participants. Their mean age was 46.1 years $(\mathrm{SD}=10.3), 31.5 \%$ were male and all were white. Pre-to-post changes in mood, perceived stress, quality of life, mindfulness skills, and introspective interest and insight were compared between the groups and the moderating and mediating effects of introspective interest and insight were examined, adjusting for effects of mindfulness skills.

Results Introspective interest nor insight moderated the effects of MBSR. However, increase in insight, but not interest, significantly mediated favorable effects of MBSR on all outcome variables, over and above changes in mindfulness skills: perceived stress $(B=-1.43,95 \% \mathrm{CI}-2.63$ to -0.54$)$, negative affect $(B=-0.11,95 \% \mathrm{CI}-0.23$ to -0.00$)$, positive affect $(B=0.12,95 \%$ CI $0.03-0.23)$ and psychological quality of life ( $B=0.16,95 \%$ CI $0.02-0.34)$.

Conclusions Increase in introspective insight may form a potentially important mechanism of the effectiveness of mindfulnessbased interventions, and possibly also other psychological interventions.
\end{abstract}

Keywords Introspection $\cdot$ Insight $\cdot$ Mediator $\cdot$ Mindfulness-based stress reduction $\cdot$ Moderator

Mindfulness-based interventions have been found to be effective in reducing symptoms of anxiety and depression across various patient and non-patient groups, with effect sizes usually around medium size in pre-post and waitlist or care-asusual control group comparisons (Khoury et al. 2013; Hofmann et al. 2010). Compared to cognitive behavioral therapy, in general, effects have been equivalent (Arch et al. 2013; Kocovski et al. 2013).

Introspection, the act of monitoring and reflecting upon inner conscious thoughts and other mental processes, has been assumed to be needed for most psychological interventions, especially insight oriented forms, to work (Joyce and McCallum 2004). Even though most clinicians and theoreticians agree with this statement, there is a paucity of empirical

Ivan Nyklíček

i.nyklicek@tilburguniversity.edu

1 Center of Research on Psychological and Somatic Disorders (CoRPS), Department of Medical and Clinical Psychology, Tilburg University, Postbox 90153, 5000 LE Tilburg, The Netherlands research regarding the role of introspection in psychological interventions, including mindfulness-based interventions (MBI). This is peculiar as an important part of many mindfulness practices is introspection: noticing internal psychological processes such as thoughts and feelings, putatively resulting in meta-cognitive insight (Chambers et al. 2009). In fact, this process has been claimed to be an important potential mechanism by which MBIs might generate their favorable effects, introspective insight facilitating cognitive defusion and decrease of negative perseverative thinking, such as rumination (Chambers et al. 2009; Nyklíček 2011). Besides introspective insight as a potential mechanism of action in MBI, a certain level of pre-intervention introspective ability has been claimed to be important for most psychological interventions to be effective, as stated above. Because introspection is part of MBI, this may also be true for this type of interventions.

In other than MBI contexts, some research has been devoted to the role of constructs in which introspection plays an important part, especially psychological mindedness (McCallum and Piper 1990b; Hall 1992) and mentalization (Bateman and Fonagy 2004). Psychological mindedness 
(PM) can be defined as a form of metacognition where a person has an interest and ability to reflect on psychological processes of both the self and others (Hall 1992; Nyklíček and Denollet 2009). Thus, the self-related part of the construct may be seen to encompass two aspects: (i) the motivation to explore one's cognitions and emotions (introspective interest), and (ii) the ability to monitor and reflect upon these processes, resulting in introspective insight. Mindfulness has a highly relevant conceptual overlap with PM (Beitel et al. 2005; Nyklíček and Denollet 2009). Mindfulness is often defined as being attentive to present moment phenomena in a nonelaborative and nonjudgmental way (Baer et al. 2006; Bishop et al. 2004). Thus, attention is open to all phenomena, including, but not being restricted to, current psychological processes. Although mindfulness encompasses more than just introspection, at the same time, mindfulness is also a more narrow concept compared to PM as it does not include elaborate reflection or evaluation of introspective processes; it just 'offers a bare display of what is taking place' (Shear and Jevning 1999).

Research using self-report scales has found PM in general to be positively associated with several adaptive person characteristics, such as openness to experience (Beitel and Cecero 2003), extraversion, conscientiousness and agreeableness (Nyklíček et al. 2010b), empathy (Beitel et al. 2005), assertiveness and sociability (Conte et al. 1995). High PM has also been related to a higher capability for dealing with ambiguity (Beitel et al. 2004). In the clinical realm, PM has mostly been viewed as a characteristic that is a prerequisite for psychotherapy to work adequately (Joyce and McCallum 2004) and consequently mainly studied as a potential moderator of adherence and treatment outcome. People high on pre-treatment PM have been found to participate in more sessions (Conte et al. 1990; Conte et al. 1996) and to drop out less (McCallum et al. 1992) compared to those low on PM. Higher PM has also been associated with higher expectations regarding therapy outcomes and with higher self-involvement in therapy in a heterogeneous patient group (Beitel et al. 2009). However, research into the role of pre-treatment PM in intervention outcomes shows inconsistent results. There has been some research, mainly from one research group, that clearly showed high pre-treatment PM to be associated with greater symptom reduction after receiving psychodynamic or supportive therapy (McCallum et al. 2003; Piper et al. 1994). However, other research showed inconsistent results within studies, where such a moderating effect of pre-treatment PM is found for some, but not other outcome measures of mostly psychodynamic therapy (Cromer and Hilsenroth 2010; Conte et al. 1990; Conte et al. 1996). In addition, several studies could not find any relation between pre-treatment PM and treatment outcomes of interventions ranging from cognitive behavioral therapy (Nyklíček et al. 2010a; Hasenauer and Tschuschke 2017) to psychodynamic therapy (Kronström et al. 2009;
Vinnars et al. 2007). Inconsistencies may stem from differences between studies, such as regarding the forms of psychotherapy used and the measuring instruments of PM. The measuring instruments all have different focusses, usually not discriminating between the facets of introspective interest and insight. However, such discrimination may be important, as will be outlined below.

Besides moderating effects of pre-treatment PM, mediating effects of increase in some facets of PM over the course of the intervention may be hypothesized to be an important ingredient for favorable effects of psychological interventions. This is based mainly on the PM component of ability to introspectively monitor and reflect on one's psychological processes, resulting in insight into one's internal dynamics (Fonagy 2006; Nyklíček et al. 2010a). However, change in PM over the course of therapy has seldom been examined, the few available studies suggesting PM to increase over the course of psychodynamic (Vinnars et al. 2009) and cognitive behavioral therapy (Nyklíček et al. 2010a). In the latter study, increase in only the insight component of PM was indeed associated with decrease of psychological symptoms.

Increase in the ability to introspect, resulting in insight into one's psychological processes has been much more strongly conceived of as being an important mechanism for favorable psychotherapy outcome in the related area of mentalization theory. Mentalization is often defined as the ability to make sense of the actions of oneself and others on the basis of intentional mental states, such as desires, feelings and beliefs (Bateman and Fonagy 2004). Especially in the area of treatment of borderline personality disorder, mentalization-based treatment is built upon the notion that enhancement of mentalization is an important working mechanism (Bateman and Fonagy 2013). Studies examining whether such enhancement indeed is responsible for treatment effects have been equivocal to date. For example, mentalization scores at the beginning of psychotherapy were correlated with global outcome at the end of treatment, but did not increase over the course of treatment (Boldrini et al. 2018) and when increases in mentalization over the course of the treatment were found, these increases were not related to outcome (Levy et al. 2006; Rudden et al. 2006).

Most of the aforementioned research has studied PM or mentalization as a single variable, while both have been conceptualized as multidimensional constructs (Fonagy 2006; McCallum and Piper 1996). The few studies that have distinguished between the different constituting components showed different associations with outcome. For instance, only increase in the introspective insight, not the interest, component of PM was found to be associated with decrease in symptoms after psychotherapy in a heterogeneous psychiatric sample (Nyklíček et al. 2010a). Thus, it is preferable to study PM as a multidimensional construct. 
Given the overlap between the introspective and mindfulness constructs and the partial focus on introspection in MBIs, the aim of the present investigation was to examine whether introspective interest and insight may contribute to outcome of a MBI, either as pre-treatment characteristics (moderators) or skills facilitated by the intervention (mediators). It was chosen to examine the role of these processes in the context of a widely used mindfulness-based intervention: mindfulnessbased stress reduction (MBSR) (Kabat-Zinn 1990). Because MBSR applies nonjudgmental acceptance instead of changing the contents of one's psychological processes (Chambers et al. 2009), it is expected that openness and interest to nonjudgmentally observe one's psychological processes may facilitate the effectiveness of this intervention. Therefore, despite the highly inconsistent previous findings regarding potential moderating effects of pre-treatment PM, in the present context of MBSR, we expect higher pre-treatment levels of introspective interest, but not necessarily insight, to predict stronger favorable changes in treatment outcome. Based on the theoretically plausible role of gaining insight into one's psychological processes in favorable intervention outcome and based on previous findings (Fonagy 2006; Nyklíček et al. 2010a), we hypothesized that increase in introspective insight, but not necessarily interest, would be associated with more favorable outcome after MBSR. In addition, the change in insight is expected to statistically mediate the effects of MBSR on treatment outcome. Both mediation and moderation effects of these facets of introspection were examined while also controlling for similar possible effects of mindfulness skills, because of such effects found in previous studies. Treatment outcome was operationalized as reduction of perceived stress and negative affect, as well as increase in positive affect and general psychological quality of life, to have a balanced negative and positive outcomes examination.

\section{Methods}

\section{Participants}

The sample used in the present study is described in a previous article (Nyklíček et al. 2013). Participants $(N=147)$ are from two highly similar sub-studies ( $N=59$ and 88 , respectively). They were recruited via advertisements in a local newspaper, which advertised for participation in a stress reduction program. Inclusion criteria were age above 18 years and answering 'regularly' or 'often' to the question 'How often would you say you feel distressed?' Exclusion criteria were insufficient knowledge of the Dutch language and serious psychopathological problems (e.g. suicidal ideation or history of psychoticism), as assessed using a self-report question during a brief paper-and-pencil intake. The study was conducted according to the Helsinki Declaration of 1975, as revised in
2000, and approved by the Medical Ethics Committee of St. Elisabeth Hospital, located in Tilburg, The Netherlands.

The power analysis was based upon previous metaanalyses on the effects of mindfulness-based interventions on psychological well-being variables in randomized trials, usually showing about a mean medium sized effect size $(d=.5)$. With an alpha level of .05 and a power of $.80,63$ participants per group were needed for the Time by Group interaction effect. Taking into account an attrition rate of $10 \%$, at least 70 participants per group were needed.

\section{Procedure}

The study was a randomized controlled trial using two parallel groups formed by balanced randomization (1:1). Participants were randomized into either the intervention group or a waitlist control group. Random selection without stratification was performed using SPSS software (procedure Select Cases) on numbers representing potential participants. The performer of this procedure (first author) received a list with numbers from a research assistant and did not know which number represented which participant. After randomization, no blinding to group assignment was possible, except for assessment of the outcomes, which was done by sending questionnaires to all participants by post by the research assistant, who also assigned participants to intervention arms.

Before the participants were randomized to the conditions, they completed the pre-treatment questionnaires and signed their informed consent at home. Subsequently, the participants in the treatment condition received a standard MBSR intervention (Kabat-Zinn 1982, 1990), which consisted of eight group sessions of $150 \mathrm{~min}$, a 6-h Sunday retreat and daily homework exercises. Groups consisted of 12 to 15 participants. During the intervention, they received psychoeducation regarding stress and mindfulness and instructions to practice mindful breathing, mindful moving (hatha yoga exercises) and sitting meditation, which they also had to practice at home (at least 40 min a day). The total treatment length was 8 weeks and was delivered for free at Tilburg University by a novice mindfulness trainer after having followed the 7-day Intensive Training at the Center for Mindfulness at Massachusetts. After the intervention period had passed, both groups received the post-treatment questionnaire to fill in and send back by mail. At the end of data collection, the participants in the waiting list group were provided with the opportunity to also receive the treatment.

\section{Measures}

Treatment outcomes included perceived stress, psychological quality of life and affective well-being. Perceived stress will be the focal point of this study, as it is the main reason for participation and the focus of MBSR. To find the unique 
effects of introspective interest and insight, mindfulness skills will be included in this study as control variables.

Perceived Stress The original Perceived Stress Scale (PSS) (Cohen et al. 1983) consists of 14 questions regarding the amount of stress the participants felt in the last month, which amounts to one perceived stress score. Items include 'How often have you been upset because of something that happened unexpectedly' and 'how often have you felt that things were going your way' (reversed scoring). Participants gave their response on a scale from 0 ('never') to 4 ('constantly/ always'). The scale has adequate reliability (Cronbach's alpha $=.84-.86)$, and discriminant and predictive validity (Cohen et al. 1983; Cohen et al. 1993). The Dutch version used in the present study also showed good reliability (Cronbach's alpha $=.87$ ) and concurrent and predictive validity (De Vries and Drent 2004). In assessing the reliability of the scale in our sample, an adequate reliability was also found $($ Cronbach's alpha $=.84)$.

Positive and Negative Affect State positive and negative affect were measured using the Global Mood Scale (GMS) (Denollet 1993) in the first cohort and the Positive and Negative Affect Schedule (PANAS) (Watson et al. 1988) in the second. Both scales consist of 20 items concerning several mood states, with 10 items reflecting positive affect (e.g. 'active', 'relaxed', 'interested') and 10 items reflecting negative affect (e.g. 'scared', 'anxious', 'guilty'). Participants used a Likert scale from 1 ('slightly or not at all') to 5 ('very much') to indicate how much they had felt this emotion recently. Both measures have been shown to have adequate reliability (Cronbach alpha $>.80$ ) and convergent and discriminant validity (Watson et al. 1988; Denollet 1993). The Dutch versions have been used, which also show good psychometric properties. The Dutch GMS showed Cronbach alpha's of .93 and .94, the Dutch PANAS of .87 and .88, while convergent validity was established by (i) intercorrelations of their respective Positive Affect scales of $r=.79$ and of their Negative Affect scales of .56, and (ii) substantial correlations $(r>.50)$ with related measures of affect (Denollet and De Vries 2006). In the present study, all four scales showed sufficient reliability with Cronbach's alpha's above .80 on both time points. As two substantially intercorrelated measures were used in the different cohorts, the scores for these scales were standardized to allow for the pooling of both cohorts, as has been done before (Nyklíček et al. 2013).

Quality of Life Quality of life was assessed using the abbreviated version of the World Health Organization Quality of Life scale (WHOQOL-BREF) (WHOQOL Group 1998). This scale, which has been simultaneously developed in 13 countries, including the Netherlands, assesses quality of life in five domains; social, environmental, physical, psychological and overall quality of life. For the purpose of this study, we examined only the psychological domain of quality of life. This is measured using 6 items regarding general psychological wellbeing and meaningfulness (e.g. 'How much do you enjoy life?' and 'To what extent do you feel your life is meaningful?'), which are answered on 5-point Likert scales. This subscale has been shown to have good construct validity and satisfactory reliability, including a Cronbach alpha of .76 across countries, including the Netherlands (WHOQOL Group 1998). In the present study, Cronbach's alpha was .75.

Mindfulness Skills The Mindful Attention Awareness Scale (MAAS) (Brown and Ryan 2003) measures the frequency a person acts mindfully in their everyday life. This facet of mindfulness may be called 'acting with awareness' or briefly 'ActAware' (Baer et al. 2006). All 15 items are formulated in a way that agreement with the statement reflects a lack of mindfulness ('I find myself doing things without paying attention'). The scale has been shown to have a Cronbach's alpha above .80 , in addition to a sufficient discriminant and convergent validity (Brown and Ryan 2003). Reliability and factorial structure have also been confirmed for the Dutch version of the scale (Schroevers et al. 2008). Reliability of the MAAS was also adequate in our sample (Cronbach's alpha $=.84$ ).

The Kentucky Inventory of Mindfulness Skills (KIMS) (Baer et al. 2004) consists of four subscales of which two were selected; the 12 item 'Observe' (Cronbach's alpha = .86-.87) and 9 item 'Accept without Judgement' (Cronbach's alpha = .89-.91) subscales. For both scales, participants indicate their response on a 5-point Likert scale from 1 ('almost never') to 5 ('almost always'). The Observe subscale assesses the awareness of sensations in the present moment ('when I'm walking, I deliberately notice the sensations of my body moving'), while the latter subscale informs on how accepting, non-judgmental, the participant is towards these sensations or thoughts with for example the reverse scored item: 'I make judgements about whether my thoughts are good or bad'. The other subscales were omitted from this study as the 'Act with Awareness' scale largely consists of items of MAAS, and the 'Describe' subscale reflects a component of mindfulness that is not primarily focused on in MBSR. The Dutch translation has shown adequate reliability and validity (Bohlmeijer et al. 2011).

Introspective Interest and Insight To assess the participant's introspective interest and insight, the Balanced Index of Psychological Mindedness (BIPM) (Nyklíček and Denollet 2009) was employed. The BIPM can be divided into two subscales: Interest and Insight, which both consist of seven items. The Interest subscale includes items like 'My attitude and feelings about things fascinate me' and reflects the amount of interest one has for internal psychological phenomena. The Insight subscale is about the ability to make sense 
and be aware of these phenomena (e.g. 'I can't make sense of my feelings', reversed scored). Responses were provided on a 5-point Likert scale from 1 ('strongly disagree') to 5 ('strongly agree'). Adequate Cronbach's alpha (.85 for Interest and .76 for Insight), test re-test reliability, convergent validity and discriminant validity were reported for the Dutch version (Nyklíček and Denollet 2009). In our sample, Cronbach's alpha was above .79 for both subscales.

\section{Data Analyses}

SPSS version 22 was used to perform statistical analyses. First, to explore basic changes over time on the psychological variables in both groups, linear mixed model analysis was applied. This analysis included only time as within-subject and group as between-subject factors, in addition to their interaction. Linear mixed model analysis has several advantages over the more conventional repeated measures ANOVA: violation of the sphericity assumption does not influence the results and values of participants with occasional missing values are still used to compute the model, reducing risk of bias due to nonrandom missingness.

Linear mixed model analysis is also used for tests of the hypotheses regarding moderation. To this end, the following variables were entered as predictors in the first model, including the potential introspective moderators and basic variables to adjust for: three-way interactions with time and group (within cohort if this would improve model fit) of age, sex, education, use of psychotropics, introspective insight and interest, as well as all lower-order two-way interactions between these variables and time and group, and all main effects. It was tested whether this model would have improved the fit indices AIC and BIC compared to the basic model including only time and group and their interaction. In a second model, the three-way interaction effects with mindful observing, accepting and acting with awareness were added, to examine if the hypothesized effect would remain significant over and beyond potential moderating effects of baseline mindfulness skills.

However, because of the multitude of effects, a third and final model was computed that was reduced to include only the three-way interaction related to the hypothesis (including introspective interest) and any other effect that was significantly contributing to the model. To account for multiple testing, interpreted were only those non-hypothesis related effects that retained significance after a Bonferroni correction within each group of effects (main effects, two-way interactions with time, two-way interactions with group, and three-way interactions), implying an alpha of .01 in the model without mindfulness skills (divided by 5) and an alpha of .0071 in the model also including the mindfulness skills.

For mediation analysis, the Preacher and Hayes approach of testing indirect effects with bootstrapping was applied
(Preacher and Hayes 2008). Specifically, the INDIRECT macro for SPSS version 4.2 was used. In this analysis, dependent variables were defined as pre-post change in the outcome variables, and mediators as pre-post change in the introspective and mindfulness variables. This analysis estimates an $a$ path (i.e. effect of condition on change in mediating variables), a $b$ path (effect of change in mediating variables on outcome variable), a total effect ( $c$ path) of condition on outcome variable and a $c^{\prime}$ path (the direct effect of condition on outcome variable controlled for the mediating variables). Finally, it estimates the indirect effects of condition through the mediator ( $a b$ path) controlled for the other mediators included in the model. These indirect effects and the $95 \%$ confidence interval are estimated with a bootstrapping approach. In the current mediation analysis, all mediating variables were simultaneously included to control for each other's effects. Here, the hypothesized mediator was change in Insight. Because there were four other potential mediators included for comparison purposes, their tests would be interpreted using a Bonferroni correction, implying an alpha of .05/ $4=.0125$, except when systematic effects of a mediator across dependent variables would warrant a discussion.

\section{Results}

All people who subscribed for the study complied with the inclusion and exclusion criteria. The final sample consisted of 143 participants of the 147 that applied. In the waiting list condition, three participants were excluded as they did not complete baseline questionnaires (remaining $N=71$ ). In the experimental condition, one participant dropped out before the intervention started (final $N=72$ ) (Fig. 1). The sample was all white and had a mean age of 46.1 years $(\mathrm{SD}=10.3$, range $21-66$ years); $45(31.5 \%)$ participants were male. Approximately half $(53.8 \%)$ of the participants received higher education (i.e. 53 received higher vocational education and 25 went to university) and $46.2 \%$ had a high school degree $(n=28)$, mid-level vocational training $(n=30)$, lower vocational training $(n=14)$ or only a primary school degree $(n=2)$. Forty-three $(30 \%)$ participants were using psychotropic medication at the start of the study.

Participants in the two cohorts did not differ on any continuous variable $(p>.10)$, gender $\left(\chi^{2}(1)=0.30, p=.583\right)$ or education $\left(\chi^{2}(1)=0.01, p=.926\right)$, except for the mindfulness subscale Observe $(F(1,139)=4.827, p=.03)$. Participants in the second cohort scored significantly higher on mindful observing compared to the first cohort (Table 1). To check whether randomization was successful, the waiting list and experimental group were compared on age, and baseline perceived stress, general quality of life, negative affect, positive affect, introspective and mindfulness skills using a 
Fig. 1 Flow diagram

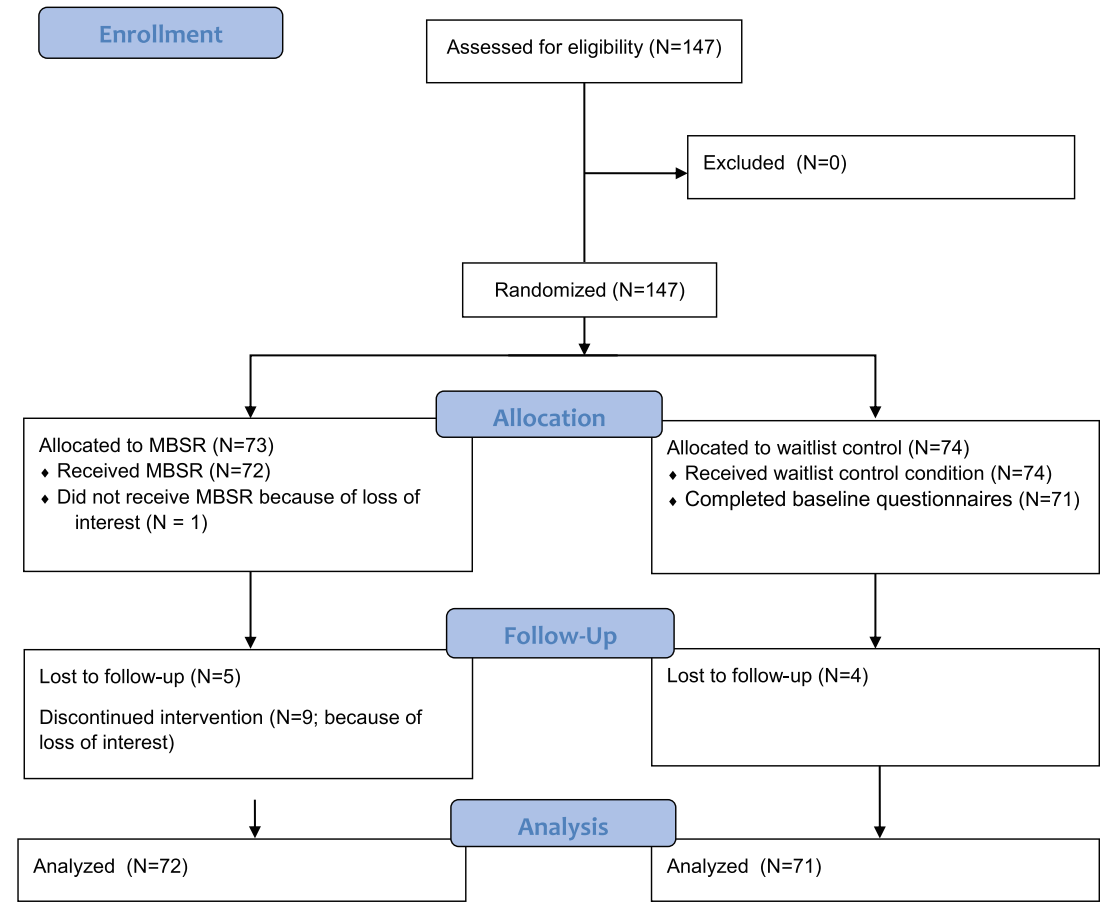

MANOVA. This revealed that the two groups did not differ on any of these variables $(p>.10)$. Neither did the two groups differ in terms of gender $\left(\chi^{2}(1)=0.84, p=.772\right)$ or education $\left(\chi^{2}(1)=2.99, p=.084\right)$.

Baseline scores on mindful observing were similar to those of two healthy American student samples but also a sample of patients with borderline personality disorder (Baer et al. 2004), all samples scoring around 37 with standard deviations of around 7 to 9 . Regarding accepting, the mean score of the present sample $(25.11, \mathrm{SD}=6.95)$ was somewhat lower compared to two healthy American student samples (30.11, SD = 6.01 and 29.61, SD=6.50), but higher compared to an American sample of patients with borderline personality disorder (21.50, SD=7.49) (Baer et al. 2004). Mean score on acting with awareness in our sample was somewhat lower (3.38, $\mathrm{SD}=0.68$ ) compared to American healthy student and cancer samples $(3.85, \mathrm{SD}=0.68$ and $4.27, \mathrm{SD}=0.64$, respectively) (Brown and Ryan 2003). Mean introspective interest and insight scores were similar to scores of a Dutch general population sample $(17.15, \mathrm{SD}=4.47$ and 19.10, $\mathrm{SD}=4.45$, respectively) and higher than those of a heterogeneous psychiatric sample (15.91, $\mathrm{SD}=5.58$ and 13.18, $\mathrm{SD}=5.65$, respectively) (Nyklíček and Denollet 2009).

Baseline correlations between mindfulness and introspective variables were nonsignificant for accepting and acting with awareness with introspective interest, while acting with awareness correlated modestly with introspective insight and observing correlated moderately with both introspective variables (Table 2).

\section{Basic Intervention Effects}

To facilitate interpretation of the hypothesis tests below, first we examined if the intervention was effective in reducing perceived stress and negative affect and increasing positive affect and psychological quality of life in the current combined sample. Compared to the control group, participants in MBSR showed a significantly larger reduction in perceived stress $\left(F(1,140)=7.58, p=.007\right.$, partial $\left.\eta^{2}=.051\right)$, negative $\operatorname{affect}\left(F(1,141)=5.96, p=.016\right.$, partial $\left.\eta^{2}=.040\right)$, and larger increase in positive affect $(F(1,141)=9.42, p=.003$, partial
Fig. 2 Indirect effects of change in introspective insight on change in perceived stress

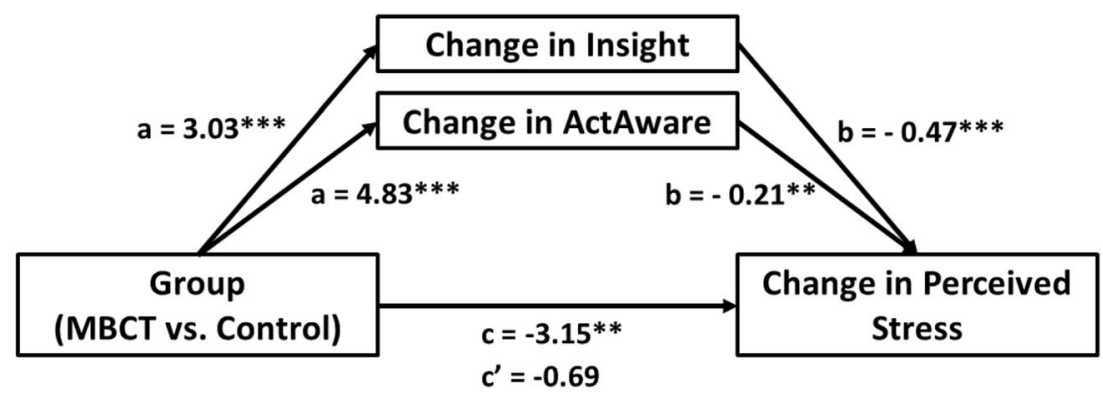


Table 1 Baseline descriptive statistics of the samples: means (standard deviations) or numbers (\%)

\begin{tabular}{|c|c|c|c|c|c|}
\hline & $\begin{array}{l}\text { Cohort } 1 \\
\text { MBSR } \\
N=29\end{array}$ & $\begin{array}{l}\text { Cohort } 1 \\
\text { Control } \\
N=28\end{array}$ & $\begin{array}{l}\text { Cohort } 2 \\
\text { MBSR } \\
N=43\end{array}$ & $\begin{array}{l}\text { Cohort } 2 \\
\text { Control } \\
N=43\end{array}$ & $p$ values \\
\hline Age & $45.8(11.6)$ & $46.2(8.4)$ & $47.4(10.8)$ & $44.6(10.2)$ & $>.10$ \\
\hline Female sex & $21(72 \%)$ & $17(61 \%)$ & $28(77 \%)$ & $33(65 \%)$ & $>.10$ \\
\hline High education & $12(41 \%)$ & $17(61 \%)$ & $21(50 \%)$ & $25(58 \%)$ & $>.10$ \\
\hline Psychotropic meds & $8(28 \%)$ & $9(29 \%)$ & $9(21 \%)$ & $17(41 \%)$ & $>.10$ \\
\hline Perceived stress & $32.80(7.07)$ & $30.99(6.13)$ & $29.00(8.12)$ & $31.10(4.93)$ & $.09^{\mathrm{a}}$ \\
\hline Quality of life & $17.34(3.49)$ & $17.61(2.96)$ & $18.21(3.07)$ & $17.64(2.53)$ & $>.10$ \\
\hline Negative affect & $0.06(0.90)$ & $-0.06(1.11)$ & $-0.22(1.05)$ & $0.22(0.90)$ & $>.10$ \\
\hline Positive affect & $-0.22(0.88)$ & $0.22(1.08)$ & $-0.02(1.17)$ & $0.02(0.80)$ & $>.10$ \\
\hline Interest & $16.76(3.80)$ & $17.63(4.50)$ & $17.88(6.24)$ & $17.42(5.84)$ & $>.10$ \\
\hline Insight & $18.45(4.75)$ & $19.41(4.85)$ & $19.58(6.92)$ & $18.30(6.65)$ & $>.10$ \\
\hline ActAware & $3.30(0.59)$ & $3.37(0.74)$ & $3.53(0.73)$ & $3.28(0.64)$ & $>.10$ \\
\hline Observing & $38.07(10.20)$ & $39.55(7.27)$ & $35.76(9.23)$ & $35.85(5.65)$ & $.03^{\mathrm{b}}$ \\
\hline Accepting & $23.69(7.31)$ & $24.38(6.25)$ & $27.42(7.05)$ & $24.24(6.83)$ & $>.10$ \\
\hline
\end{tabular}

${ }^{a}$ Group $\times$ Cohort interaction, ${ }^{b}$ main effect of cohort; ActAware acting with awareness (scores on the Mindful Attention Awareness Scale)

$\left.\eta^{2}=.063\right)$ and psychological quality of life $(F(1,139)=8.37$, $p=.004$, partial $\eta^{2}=.057$ ) (Table 3).

They also showed significantly larger increases in the mindfulness facets acting with awareness $(F(1,141)=$ 13.01, $p<.001$, partial $\left.\eta^{2}=.084\right)$, accepting $(1,141)=6.15$, $p=.014$, partial $\left.\eta^{2}=.042\right)$, and observing $(F(1,141)=27.57$, $p<.001$, partial $\left.\eta^{2}=.164\right)$. Importantly, introspective insight $\left(F(1,138)=17.46, p<.001\right.$, partial $\left.\eta^{2}=.112\right)$ also showed a larger increase in the MBSR group, while there was no differential change observed in introspective interest $(F(1,138)=$ 2.20, $p=.141$, partial $\eta^{2}=.016$ ) (Table 3).

\section{Pre-treatment Moderators of Outcome}

First, linear mixed models including time, group (within cohort if showing a better fit), sex, age, education, use of psychotropics, introspective interest and insight and their interactions with group and time were performed to examine the hypothesized moderating effect of introspective interest, compared to the effect of introspective insight and controlled for such effects of basic demographic characteristics and use of psychotropics.

For perceived stress, analyses revealed no significant threeway interaction effects with group (within cohort) and time, indicating no moderation. The model showing the best fit $(-2$ $\log$ likelihood $=1696$; $\mathrm{AIC}=1702 ; \mathrm{BIC}=1712$ ) was significantly better $(p<.001)$ than the basic model including only the Time $\times$ Group interaction effects. The Group $\times$ Time $\times$ Interest interaction effect was not significant $(F(3,133)=$ $2.38, p=.073$ ) (Table 4). Of the control variables, only significant Bonferroni adjusted main effects were found for baseline interest and mindful accepting, both showing negative overall

Table 2 Pearson correlations between baseline mindfulness, introspection and outcome variables

\begin{tabular}{|c|c|c|c|c|c|c|}
\hline & Perceived stress & Quality of life & Negative affect & Positive affect & Interest & Insight \\
\hline Perceived stress & & $-.61 * * *$ & $.64 * * *$ & $-.58 * * *$ & $-.24 * *$ & $-.26^{* *}$ \\
\hline Quality of life & & & $-.55 * * *$ & $.54 * * *$ & $.25 * *$ & $.27^{* *}$ \\
\hline Negative affect & & & & $-.30 * *$ & -.09 & -.05 \\
\hline Positive affect & & & & & $.25 * *$ & $.38 * *$ \\
\hline Interest & & & & & & $.39 * *$ \\
\hline \multicolumn{7}{|l|}{ Insight } \\
\hline ActAware & $-.26^{* *}$ & $.31 * *$ & $-.32 * *$ & $.18^{*}$ & .09 & $.24 * *$ \\
\hline Observing & -.13 & $.20 *$ & -.02 & $.28 * *$ & $.46^{* *}$ & $.44 * *$ \\
\hline Accepting & $-.32 * *$ & $.33 * *$ & $-.28 * *$ & $.21 *$ & -.11 & .04 \\
\hline
\end{tabular}

$* * * p<.001 ; * * p<.01 ; * p<.05 ; \# p<.10 ;$ ActAware acting with awareness (scores on the Mindful Attention Awareness Scale) 
Table 3 Mean scores of psychological variables per time point, including test of time by group interaction

\begin{tabular}{|c|c|c|c|c|c|}
\hline & \multicolumn{2}{|c|}{ Waiting list $(n=71)$} & \multicolumn{2}{|l|}{$\operatorname{MBSR}(n=72)$} & \multirow{2}{*}{$\begin{array}{l}\text { Interaction } \\
\text { Partial } \eta^{2}\end{array}$} \\
\hline & Time $1 \mathrm{M}(\mathrm{SD})$ & Time 2 M(SD) & Time $1 \mathrm{M}(\mathrm{SD})$ & Time2 M(SD) & \\
\hline ActAware & $3.32(0.68)$ & $3.33(0.65)$ & $3.44(0.68)$ & $3.76(0.63)$ & $.084 * * *$ \\
\hline Observing & $37.31(6.54)$ & $36.41(7.04)$ & $36.69(9.63)$ & $40.36(7.68)$ & $.164 * * *$ \\
\hline Accepting & $24.29(6.56)$ & $25.33(6.53)$ & $25.92(7.34)$ & $29.07(6.58)$ & $.042 *$ \\
\hline Insight & $18.84(5.97)$ & $18.12(5.54)$ & $19.20(6.14)$ & $21.47(5.35)$ & $.112 * * *$ \\
\hline Interest & $17.51(5.37)$ & $17.54(5.26)$ & $17.50(5.39)$ & $18.39(5.04)$ & .016 \\
\hline Perceived stress & $31.06(5.39)$ & $28.54(6.99)$ & $30.40(7.87)$ & $24.77(8.49)$ & $.051 * *$ \\
\hline Psychol QoL & $17.58(2.68)$ & $18.22(3.32)$ & $17.86(3.25)$ & $19.51(3.06)$ & $.057^{* *}$ \\
\hline Negative affect & $0.11(0.99)$ & $0.27(0.99)$ & $-0.11(1.00)$ & $-0.26(0.94)$ & $.040 *$ \\
\hline Positive affect & $0.10(0.92)$ & $-0.12(0.87)$ & $-0.10(1.06)$ & $0.12(1.10)$ & $.063 * *$ \\
\hline
\end{tabular}

$* * * p<.001 ; * * p<.01 ; * p<.05 ;$ ActAware acting with awareness (scores on the Mindful Attention Awareness Scale); Psychol QoL psychological quality of life

associations with perceived stress; coefficients being -0.86 and -0.30 , respectively, both $p<.001$ (Table 4). No significant three-way interactions with time and group were obtained for baseline mindfulness skills.

For psychological quality of life, the model showing the best fit $(-2 \log$ likelihood $=1206 ; \mathrm{AIC}=1254 ; \mathrm{BIC}=1341)$ did not include cohort and was significantly better $(p<.001)$ than the basic model including only the Time $\times$ Group interaction effects. No three-way interaction effects with group and time or two-way interactions with time appeared, except the original Time $\times$ Group interaction $(F(1,140)=12.21$, $p<.001)$. Of the control variables, the only Bonferroni corrected significant effects were main effects of mindful accepting and education; of both variables, higher levels were associated with overall higher psychological quality of life (coefficients $=0.14, p<.001$, and $1.17, p=.005$, respectively). No significant three-way interactions with time and group were obtained for baseline mindfulness skills.

Regarding negative and positive affect, no significant three-way interaction with group and time appeared, although the best models (not including cohort) showed improved fit (both $p<.001$ ) compared to the basic model (for negative

Table 4 Predictors (hypothesized are in italics) of change in outcome variables: $F$ values of fixed effects in mixed linear models analysis

\begin{tabular}{|c|c|c|c|c|}
\hline & Perceived stress & Quality of life & Negative affect & Positive affect \\
\hline Time & & $44.63 * * *$ & $3.88 \#$ & \\
\hline Group (within cohort) & $3.18^{*}$ & & $1.57 \#$ & \\
\hline Education & & $8.24 * *$ & & $7.77 * *$ \\
\hline Accepting & $26.90 * * *$ & $36.10 * * *$ & $16.73 * * *$ & $20.50 * * *$ \\
\hline Interest & $21.95 * * *$ & $3.77 \#$ & $6.23 *$ & \\
\hline Insight & & $4.81 *$ & & $15.09 * * *$ \\
\hline Group $\times$ time & 1.66 & $12.21 * * *$ & $8.92 * *$ & $7.72 * * *$ \\
\hline Age ×time & & & $3.06 \#$ & \\
\hline Interest $\times$ time & 1.76 & & & \\
\hline Interest $\times$ group & $4.17 * *$ & & & \\
\hline Observe×group & & $7.39 * *$ & & \\
\hline Insight $\times$ group & & $5.13 *$ & & \\
\hline Interest $\times$ time $\times$ group & $2.38 \#$ & & 1.62 & \\
\hline
\end{tabular}

Effects included in the complete models were the variables group (nested within cohort for perceived stress), time, age, sex, education and use of psychotropics, Interest, Insight, observing, accepting and acting with awareness, and including all two- and three-way interaction effects with group and time. Depicted here are the effects remaining in the final model including only the hypothesized three-way interaction (Interest $\times$ Time $\times$ Group) and its lower-order interactions and main effects and any other significant effects $* * * p<.001 ; * * p<.01 ; * p<.05 ; \# p<.10$ (not Bonferroni corrected) 
affect $-2 \log$ likelihood $=707$; $\mathrm{AIC}=721$; $\mathrm{BIC}=747$; for positive affect $-2 \log$ likelihood $=673$; $\mathrm{AIC}=695 ; \mathrm{BIC}=735$ ). The only significant interaction effect with time and group included only these two variables as in the basic model (Table 4). The only control variables that were Bonferroni corrected significant were main effects of baseline mindful accepting, showing overall associations with negative affect $(-0.03, p<.001)$ and positive affect $(0.03, p<.001)$, introspective insight, showing an overall positive association with positive affect $(0.04, p<.001)$, and education, also showing an overall positive association with positive affect $(0.35$, $p=.006$ ) (Table 4). No significant three-way interactions with time and group were obtained for baseline mindfulness skills.

\section{Mediators of Outcome}

The indirect effects test macro for SPSS by Preacher and Hayes with bootstrapping included all introspective and mindfulness variables in every model to control for each other's effects. In line with the results regarding intervention effects shown above, the total effects of the intervention ( $c$ path) were significant for all outcome variables (Table 5). Also the effects of the intervention on the mindfulness and introspective mediators were significant, except on Interest ( $a$ paths). Associations between the potential mediators and outcome variables ( $b$ paths) were found mainly for increase in introspective insight (significant for all outcomes), and increase in acting with awareness (significant for changes in perceived stress, quality of life and negative affect).

These significant $b$ paths were also largely reflected in mediating indirect effects ( $a b$ paths), which were also found for increase in insight for all outcome variables and for acting with awareness for changes in perceived stress, quality of life and negative affect (Table 5) (Fig. 2 for illustration regarding perceived stress).

The mediators statistically fully mediated the effects on perceived stress $\left(\mathrm{c}^{\prime}=-0.69(t(139)=-0.55, p=.581)\right)$, quality of life $\left(c^{\prime}=0.09(t(132)=.34, p=.734)\right.$, negative affect $\left(c^{\prime}=-0.21, t(133)=-1.45, p=.150\right)$ and positive affect $\left(c^{\prime}=0.08(t(133)=0.49, p=.627)\right.$.

\section{Discussion}

Introspection, resulting in insight, has long been claimed to be an important process in psychological interventions (Appelbaum 1973; Hall 1992), but its role has been understudied. Therefore, the aim of the present study was to examine the role of introspective interest and insight in the context of a mindfulness-based stress reduction intervention. Both moderator and mediator were examined, adjusting for similar effects of mindfulness skills. Given the conceptual difference between these two facets of introspective capacities, interest in one's psychological phenomena may be particularly expected to be a kind of a prerequisite for psychological interventions to work (McCallum and Piper 1990a; Conte et al. 1990), while increase in insight into these phenomena may be expected to be a mechanism mediating favorable effects of interventions (Hall 1992; Nyklíček et al. 2010a). Mindfulness-based interventions offer an outstanding opportunity for such test, as introspection is a central facet of these interventions (Kabat-Zinn 1990).

No moderator effects were found for pre-treatment introspective interest or insight on any of the outcome variables. This finding is in line with previous research in other psychological interventions, showing no moderating effects of these variables on psychological outcomes (Nyklíček et al. 2010a). Research on related variables such as mentalization and differently operationalized psychological mindedness also often showed no effects or inconsistent results (Cromer and Hilsenroth 2010; Kronström et al. 2009).

While it is possible that pre-treatment levels do not play an important role, it may also be the case that self-selection bias of individuals relatively high in pre-treatment levels of these introspective qualities might have restricted the range of values to the higher end, making associations difficult to obtain. Indeed, in the present study, pre-treatment levels of both introspective interest and insight were higher compared to those of a sample of psychiatric patients and similar to those of a healthy Dutch population sample (Nyklíček and Denollet 2009). Therefore, it seems worthwhile to examine such possible moderator effects in samples of people who have not proactively searched for a mindfulness intervention, but receive such intervention as part of their treatment plan.

In these analyses, a consistent finding across dependent variables was the main effect of pre-treatment levels of the mindfulness skill of accepting, showing positive associations with positive affect and psychological quality of life and negative associations with negative affect and perceived stress across time. Such effect might have been anticipated, as it is in line with both theory implicating the importance of acceptance for stress reduction (Baer et al. 2004; Kabat-Zinn 1990) and previous findings showing acceptance to facilitate coping with adverse situations (Pakenham and Samios 2013). A similar effect was obtained for pre-treatment levels of introspective insight regarding positive affect and to a lesser extent psychological quality of life. This may indicate a similar general adaptive quality of insight, which is in line with crosssectional negative correlations between levels of insight and symptoms of psychopathology (Nyklíček and Denollet 2009).

Regarding mediation, conform our hypothesis, increase in introspective insight statistically mediated the effects of MBSR on all outcome variables. This was found even when controlled for similar mediation effects of increase in mindfulness skills. Outcome variables were stress and negative affect as well as psychological quality of life and positive affect. This suggests that introspective insight may indeed be 
Table 5 Direct and indirect mediating effects of change for the hypothesized (in italics) and other effects

\begin{tabular}{|c|c|c|c|c|c|c|c|}
\hline & \multicolumn{2}{|l|}{ a path } & \multicolumn{2}{|l|}{ b path } & \multicolumn{3}{|l|}{ ab path } \\
\hline & $B$ & SE & $B$ & SE & $B$ & SE & $95 \% \mathrm{CI}[\mathrm{LB}, \mathrm{UB}]$ \\
\hline \multicolumn{8}{|c|}{$\Delta$ Perceived stress } \\
\hline$\Delta$ ActAware & $4.83^{*}$ & 1.28 & $-0.21^{*}$ & 0.08 & $-0.94 *$ & 0.51 & {$[-2.22,-0.19]$} \\
\hline$\Delta$ Observing & $5.57 *$ & 1.07 & 0.02 & 0.09 & 0.13 & 0.55 & {$[-0.96,1.23]$} \\
\hline$\Delta$ Accepting & $2.50 *$ & 1.02 & -0.03 & 0.10 & -0.11 & 0.37 & {$[-0.94,0.58]$} \\
\hline$\Delta$ Insight & $3.03 *$ & 0.72 & $-0.47 *$ & 0.13 & $-1.43^{*}$ & 0.53 & {$[-2.63,-0.54]$} \\
\hline$\Delta$ Interest & 0.80 & 0.58 & -0.14 & 0.16 & -0.11 & 0.19 & {$[-0.74,0.11]$} \\
\hline \multicolumn{8}{|l|}{$\Delta$ Quality of life } \\
\hline$\Delta$ ActAware & $4.77 *$ & 1.29 & $0.03^{*}$ & 0.02 & $0.15^{*}$ & 0.07 & {$[0.03,0.34]$} \\
\hline$\Delta$ Observing & $5.29 *$ & 1.05 & 0.00 & 0.02 & 0.01 & 0.10 & {$[-0.18,0.23]$} \\
\hline$\Delta$ Accepting & $2.77 *$ & 1.04 & 0.02 & 0.02 & 0.04 & 0.05 & {$[-0.05,0.19]$} \\
\hline$\Delta$ Insight & $2.97 *$ & 0.72 & $0.06^{*}$ & 0.03 & $0.16^{*}$ & 0.07 & {$[0.02,0.34]$} \\
\hline$\Delta$ Interest & 0.77 & 0.58 & 0.00 & 0.03 & 0.00 & 0.03 & {$[-0.04,0.08]$} \\
\hline \multicolumn{8}{|c|}{$\Delta$ Negative affect } \\
\hline$\Delta$ ActAware & $4.70 *$ & 1.28 & $-0.02 *$ & 0.01 & $-0.09^{*}$ & 0.05 & {$[-0.20,-0.02]$} \\
\hline$\Delta$ Observing & $5.48 *$ & 1.06 & 0.01 & 0.01 & 0.04 & 0.07 & {$[-0.10,0.19]$} \\
\hline$\Delta$ Accepting & $2.69 *$ & 1.03 & 0.01 & 0.01 & 0.02 & 0.04 & {$[-0.04,0.14]$} \\
\hline$\Delta$ Insight & $2.99 *$ & 0.72 & $-0.04^{*}$ & 0.02 & $-0.11 *$ & 0.06 & {$[-0.23,-0.00]$} \\
\hline$\Delta$ Interest & 0.86 & 0.58 & 0.00 & 0.02 & -0.00 & 0.02 & {$[-0.03,0.06]$} \\
\hline \multicolumn{8}{|l|}{$\Delta$ Positive affect } \\
\hline$\Delta$ ActAware & $4.70 *$ & 1.28 & 0.01 & 0.01 & 0.06 & 0.05 & {$[-0.03,0.17]$} \\
\hline$\Delta$ Observing & $5.48 *$ & 1.06 & 0.02 & 0.01 & $0.11 *$ & 0.06 & {$[0.00,0.24]$} \\
\hline$\Delta$ Accepting & $2.69 *$ & 1.03 & 0.02 & 0.01 & 0.04 & 0.04 & {$[-0.01,0.14]$} \\
\hline$\Delta$ Insight & $2.99 *$ & 0.72 & $0.04 *$ & 0.02 & $0.12 *$ & 0.05 & {$[0.03,0.23]$} \\
\hline$\Delta$ Interest & 0.86 & 0.58 & $0.05 *$ & 0.02 & 0.04 & 0.04 & {$[-0.00,0.14]$} \\
\hline
\end{tabular}

$* p<.05$. Coefficients from the ab bath are bootstrapped; $\Delta=$ pre-post change; ActAware acting with awareness (scores on the Mindful Attention Awareness Scale) an important mechanism of the beneficial effects of MBSR on stress reduction and well-being enhancement. However, this suggestion has to be tested in future research with change in the mediators taking place before change in the outcome measures, to be able to draw any conclusions in the direction of mechanistic explanations. Nevertheless, the present outcome indicates the added value of including introspective variables in this field of research and discriminating between components of PM, not using PM as a unitary construct as has previously been done (Nyklíček and Denollet 2009).

In addition, these results reflecting mediating effects of introspective insight are in line with similar results in studies on dynamic psychotherapy for anxiety related personality disorders (Kallestad et al. 2010) and cognitive behavioral therapy in a heterogeneous psychiatric sample (Nyklíček et al. 2010a). This indicates that introspective insight might be an important transdiagnostic and transintervention factor in various forms of psychotherapy and should receive more attention of researchers.

In the context of mindfulness-based interventions, it is clear that introspective insight may be important, as introspection is an important part of mindfulness practices and it provides insight into important mind processes, both maladaptive and adaptive. Some examples are the nonfactual nature of thoughts, including judgments, the fluctuating nature of feelings and the insight of being able to decenter from those phenomena in a helpful (self-compassionate) way. Insight may therefore be regarded as a higher-order result of mindfulness practice, leading to a cascade of lower-order beneficial effects of mindfulness (Nyklíček 2011). This may be reflected by the facts that, in the present study, increase in insight was the only factor showing mediating effects on all outcome variables, even adjusting for effects of mindfulness skills.

Of the mindfulness skills, acting with awareness (Baer et al. 2006) was the main other factor showing mediating effects on three out of four outcome variables. The importance of acting with awareness in this study is supported by similar previous findings (Bränström et al. 2010; Nyklíček and Kuijpers 2008).

Regarding the conceptual and statistical overlap between the introspection and mindfulness constructs, it can be noted that the largest overlap was found with mindful observing, 
showing moderate correlations with both introspection variables. Statistically, the correlation showed shared variance of less than $25 \%$. On the content level, three quarters of the observing items are about noticing sensory phenomena, not psychological ones. Therefore, both the interest and insight components have added value regarding awareness of own psychological processes. Finally, the fact that change in the insight component, but not the observing scale mediated the effects on all outcome variables also demonstrates the added value of introspective insight to the value of specific mindfulness skills.

\section{Limitations and Future Research}

This study is not without its limitations. First, the selfselection of participants from the general population reacting to an advertisement for a mindfulness-based stress reduction intervention implies potential low generalizability to samples which are offered the intervention as part of their treatment plan. Such samples may show lower initial interest and openness regarding introspection, influencing the results in an unknown way. In addition, most participants were highly educated white women, further limiting generalizability. Conversely, as almost onethird of the participants were using psychotropic medication at the time of the study, this may suggest partial generalizability to clinically relevant populations. Second, the fact that the data collection has been performed some time ago has resulted in the inclusion of an older mindfulness questionnaire, which does not include the important facet of non-reactivity (Baer et al. 2006). Non-reactivity has been shown in previous research to mediate some of the mindfulness intervention effects (Labelle et al. 2015; Joseffson et al. 2011). Inclusion of this facet may have changed the results. Currently, undergoing another psychological intervention was also not assessed in the present study. The potential influence thereof on the results, independent of the use of psychotropics, would be most likely limited as the current trial was randomized. Third, the assessment of both mediating and outcome variables at the same moments does not permit any conclusions in the direction of causal explanations. Fourth, waitlist as a control group does not permit any conclusions regarding the specificity of the effects for the current intervention. Inclusion of active control groups is necessary in future research. The relative inexperience of the MBSR trainer in the current study may have limited the effects found. However, the effect sizes found regarding outcomes were comparable to those of previous MBSR studies, which suggests that the potential trainer effect was not substantial.

Future research should also include outcomes and mediators assessed by means other than self-report (significant other, behavioral tests) to reduce the risk of social desirability effects inherent in self-reports. Furthermore, examining the importance of introspective interest and insight in mindfulness-based interventions relative to the full range of mindfulness skills, as well as examining their conceptual and temporal dynamic interrelationships would be of considerable interest. Dismantling studies, in which interventions based on only one of the proposed mechanisms are needed to assess their relative importance regarding their efficacy. In addition, to test causality, future research needs experimental research specifically enhancing introspective insight and trials using more frequent assessments to be able to test temporal dynamics of change of mediators and outcomes. After such studies are conducted, statements regarding clinical implications can be made, such as to what extent it would be desirable to monitor change in insight during the course of interventions in order to use this information for client specific adjustments to the intervention.

Author Contributions IN: designed and executed the study, assisted with data analysis and wrote the manuscript. RZ: performed data analysis, wrote a first draft of the manuscript and edited latter drafts. JD: collaborated in the writing and editing of the manuscript. All authors approved the final version of the manuscript for submission.

\section{Compliance with Ethical Standards}

Conflict of Interest The authors declare that they have no conflict of interest. It may be noted that the first author is a mindfulness trainer at center Dit Moment, Tilburg, Netherlands.

Ethics Statement The study protocol of the current study was approved by the Medical Ethics Committee of St. Elisabeth Hospital, located in Tilburg, The Netherlands.

Open Access This article is licensed under a Creative Commons Attribution 4.0 International License, which permits use, sharing, adaptation, distribution and reproduction in any medium or format, as long as you give appropriate credit to the original author(s) and the source, provide a link to the Creative Commons licence, and indicate if changes were made. The images or other third party material in this article are included in the article's Creative Commons licence, unless indicated otherwise in a credit line to the material. If material is not included in the article's Creative Commons licence and your intended use is not permitted by statutory regulation or exceeds the permitted use, you will need to obtain permission directly from the copyright holder. To view a copy of this licence, visit http://creativecommons.org/licenses/by/4.0/.

\section{References}

Appelbaum, S. A. (1973). Psychological-mindedness: word, concept and essence. International Journal of Psychoanalysis, 54(1), 35-46.

Arch, J. J., Ayers, C. R., Baker, A., Almklov, E., Dean, D. J., \& Craske, M. G. (2013). Randomized clinical trial of adapted mindfulnessbased stress reduction versus group cognitive behavioral therapy for heterogeneous anxiety disorders. Behaviour Research and Therapy, 51(4-5), 185-196. 
Baer, R. A., Smith, G. T., \& Allen, K. B. (2004). Assessment of mindfulness by self-report: the Kentucky Inventory of Mindfulness Skills. Assessment, 11(3), 191-206. https://doi.org/10.1177/ 1073191104268029

Baer, R. A., Smith, G. T., Hopkins, J., Krietemeyer, J., \& Toney, L. (2006). Using self-report assessment methods to explore facets of mindfulness. Assessment, 13(1), 27-45. https://doi.org/10.1177/ 1073191105283504.

Bateman, A., \& Fonagy, P. (2004). Mentalization-based treatment of BPD. Journal of Personality Disorders, 18(1), 36-51.

Bateman, A., \& Fonagy, P. (2013). Mentalization-based treatment. Psychoanalytic Inquiry, 33(6), 595-613.

Beitel, M., \& Cecero, J. J. (2003). Predicting psychological mindedness from personality style and attachment security. Journal of Clinical Psychology, 59(1), 163-172.

Beitel, M., Ferrer, E., \& Cecero, J. J. (2004). Psychological mindedness and cognitive style. Journal of Clinical Psychology, 60(6), 567-582.

Beitel, M., Ferrer, E., \& Cecero, J. J. (2005). Psychological mindedness and awareness of self and others. Journal of Clinical Psychology, 61(6), 739-750.

Beitel, M., Hutz, A., Sheffield, K. M., Gunn, C., Cecero, J. J., \& Barry, D. T. (2009). Do psychologically-minded clients expect more from counselling? Psychology and Psychotherapy-Theory Research and Practice, 82(4), 369-383.

Bishop, S. R., Lau, M. A., Shapiro, S., Carlson, L., Anderson, N. D., Carmody, J., et al. (2004). Mindfulness: a proposed operational definition. Clinical Psychology: Science and Practice, 11(3), 230241.

Bohlmeijer, E., ten Klooster, P. M., Fledderus, M., Veehof, M., \& Baer, R. (2011). Psychometric properties of the five facet mindfulness questionnaire in depressed adults and development of a short form. Assessment, 18(3), 308-320. https://doi.org/10.1177/ 1073191111408231.

Boldrini, T., Nazzaro, M. P., Damiani, R., Genova, F., Gazzillo, F., \& Lingiardi, V. (2018). Mentalization as a predictor of psychoanalytic outcome: an empirical study of transcribed psychoanalytic sessions through the lenses of a computerized text analysis measure of reflective functioning. Psychoanalytic Psychology, 35(2), 196-204.

Bränström, R., Kvillemo, P., Brandberg, Y., \& Moskowitz, J. T. (2010). Mindfulness as a mediator of psychological well-being in a stress reduction intervention for cancer patients: a randomized study. Annals of Behavioral Medicine, 39, 151-161.

Brown, K. W., \& Ryan, R. M. (2003). The benefits of being present: mindfulness and its role in psychological well-being. Journal of Personality and Social Psychology, 84(4), 822-848. https://doi. org/10.1037/0022-3514.84.4.822.

Chambers, R., Gullone, E., \& Allen, N. B. (2009). Mindful emotion regulation: an integrative review. Clinical Psychology Review, 29(6), 560-572. https://doi.org/10.1016/j.cpr.2009.06.005.

Cohen, S., Kamarck, T., \& Mermelstein, R. (1983). A global measure of perceived stress. Journal of Health and Social Behavior, 24(4), 385-396.

Cohen, S., Tyrrell, D. A., \& Smith, A. P. (1993). Negative life events, perceived stress, negative affect, and susceptibility to the common cold. Journal of Personality and Social Psychology, 64(1), 131140.

Conte, H. R., Plutchik, R., Jung, B. B., Picard, S., Karasu, T. B., \& Lotterman, A. (1990). Psychological mindedness as a predictor of psychotherapy outcome: a preliminary report. Comprehensive Psychiatry, 31(5), 426-431.

Conte, H. R., Buckley, P., Picard, S., \& Karasu, T. B. (1995). Relationships between psychological mindedness and personality traits and ego functioning: validity studies. Comprehensive Psychiatry, 36(1), 11-17.

Conte, H. R., Ratto, R., \& Karasu, T. B. (1996). The psychological mindedness scale: factor structure and relationship to outcome of psychotherapy. Journal of Psychotherapy Practice and Research, 5(3), 250.

Cromer, T. D., \& Hilsenroth, M. J. (2010). Patient personality and outcome in short-term psychodynamic psychotherapy. Journal of Nervous and Mental Disease, 198(1), 59-66.

De Vries, J., \& Drent, M. (2004). Relationship between perceived stress and sarcoidosis in a Dutch patient population. Sarcoidosis Vasculitis and Diffuse Lung Diseases, 21(1), 57-63.

Denollet, J. (1993). Emotional distress and fatigue in coronary heart disease: the Global Mood Scale (GMS). Psychological Medicine, 23(1), 111-121.

Denollet, J., \& De Vries, J. (2006). Positive and negative affect within the realm of depression, stress and fatigue: the two-factor distress model of the Global Mood Scale (GMS). Journal of Affective Disorders, 91(2-3), 171-180. https://doi.org/10.1016/j.jad.2005.12.044.

Fonagy, P. (2006). The mentalization-focused approach to social development. In J. G. Allen \& P. Fonagy (Eds.), Handbook of mentalization-based treatment (pp. 53-100). Chichester: Wiley.

Hall, J. A. (1992). Psychological-mindedness: a conceptual model. American Journal of Psychotherapy, 46(1), 131-140.

Hasenauer, M., \& Tschuschke, V. (2017). Effectiveness and outcome prediction of outpatient cognitive behavioral therapy. Verhaltenstherapie, 27(2), 86-95.

Hofmann, S. G., Sawyer, A. T., Witt, A. A., \& Oh, D. (2010). The effect of mindfulness-based therapy on anxiety and depression: a metaanalytic review. Journal of Consulting and Clinical Psychology, 78(2), 169-183. https://doi.org/10.1037/a0018555.

Joseffson, T., Larsman, P., Broberg, A. G., \& Lund, L.-G. (2011). Selfreported mindfulness mediates the relation between meditation experience and psychological well-being. Mindfulness, 2, 49-58.

Joyce, A. S., \& McCallum, M. (2004). Assessing patient capacities for therapy: psychological-mindedness and quality of object relations. Mahwah: Lawrence Erlbaum Associates Publishers.

Kabat-Zinn, J. (1982). An outpatient program in behavioral medicine for chronic pain patients based on the practice of mindfulness meditation: theoretical considerations and preliminary results. General Hospital Psychiatry, 4(1), 33-47.

Kabat-Zinn, J. (1990). Full catastrophe living: using the wisdom of your body and mind to face stress, pain, and illness. New York: Delacourt.

Kallestad, H., Valen, J., McCullough, L., Svartberg, M., Hoglend, P., \& Stiles, T. C. (2010). The relationship between insight gained during therapy and long-term outcome in short-term dynamic psychotherapy and cognitive therapy for cluster $\mathrm{C}$ personality disorders. Psychotherapy Research, 20(5), 526-534.

Khoury, B., Lecomte, T., Fortin, G., Masse, M., Therien, P., Bouchard, V., et al. (2013). Mindfulness-based therapy: a comprehensive metaanalysis. Clinical Psychology Review, 33(6), 763-771.

Kocovski, N. L., Fleming, J. E., Hawley, L. L., Huta, V., \& Antony, M. M. (2013). Mindfulness and acceptance-based group therapy versus traditional cognitive behavioral group therapy for social anxiety disorder: a randomized controlled trial. Behaviour Research and Therapy, 51(12), 889-898.

Kronström, K., Salminen, J. K., Hietala, J., Kaijander, J., Vahlberg, T., Markkula, J., et al. (2009). Does defense style or psychological mindedness predict treatment response in major depression? Depression and Anxiety, 26(7), 689-695.

Labelle, L. E., Campbell, T. S., Faris, P., \& Carlson, L. E. (2015). Mediators of mindfulness-based stress reduction (MBSR): assessing the timing and sequence of change in cancer patients. Journal of Clinical Psychology, 71(1), 21-40.

Levy, K. N., Meehan, K. B., Kelly, K. M., Reynoso, J. S., Weber, M., Clarkin, J. F., et al. (2006). Change in attachment patterns and reflective function in a randomized control trial of transferencefocused psychotherapy for borderline personality disorder. Journal of Consulting and Clinical Psychology, 74(6), 1027-1040. 
McCallum, M., \& Piper, W. E. (1990a). A controlled study of effectiveness and patient suitability for short-term group psychotherapy. International Journal of Group Psychotherapy, 40(4), 431-452.

McCallum, M., \& Piper, W. E. (1990b). The psychological mindedness assessment procedure. Psychological Assessment, 2(4), 412.

McCallum, M., \& Piper, W. E. (1996). Psychological mindedness. Psychiatry, 59(1), 48-64.

McCallum, M., Piper, W. E., \& Joyce, A. S. (1992). Dropping out from short-term group therapy. Psychotherapy: Theory, Research, Practice, Training, 29(2), 206.

McCallum, M., Piper, W. E., Ogrodniczuk, J. S., \& Joyce, A. S. (2003). Relationships among psychological mindedness, alexithymia and outcome in four forms of short-term psychotherapy. Psychology and Psychotherapy: Theory, Research and Practice, 76(Pt 2), 133-144.

Nyklíček, I. (2011). Mindfulness, emotion regulation, and well-being. In I. Nyklíček, A. Vingerhoets, \& M. Zeelenberg (Eds.), Emotion regulation and well-being (pp. 101-118). New York: Springer.

Nyklíček, I., \& Denollet, J. (2009). Development and evaluation of the balanced index of psychological mindedness (BIPM). Psychological Assessment, 21(1), 32-44. https://doi.org/10.1037/ a0014418.

Nyklíček, I., \& Kuijpers, K. F. (2008). Effects of mindfulness-based stress reduction intervention on psychological well-being and quality of life: is increased mindfulness indeed the mechanism? Annals of Behavioral Medicine, 35(3), 331-340. https://doi.org/10.1007/ s12160-008-9030-2.

Nyklíček, I., Majoor, D., \& Schalken, P. A. (2010a). Psychological mindedness and symptom reduction after psychotherapy in a heterogeneous psychiatric sample. Comprehensive Psychiatry, 51(5), 492496. https://doi.org/10.1016/j.comppsych.2010.02.004.

Nyklíček, I., Poot, J. C., \& van Opstal, J. (2010b). Psychological mindedness in relation to personality and coping in a sample of young adult psychiatric patients. Journal of Clinical Psychology, 66(1), 34-45. https://doi.org/10.1002/jclp.20627.

Nyklíček, I., van Beugen, S., \& Denollet, J. (2013). Effects of mindfulness-based stress reduction on distressed (type D) personality traits: a randomized controlled trial. Journal of Behavioral Medicine, 36, 361-370. https://doi.org/10.1007/s10865-012-94313.

Pakenham, K. I., \& Samios, C. (2013). Couples coping with multiple sclerosis: a dyadic perspective on the roles of mindfulness and acceptance. Journal of Behavioral Medicine, 36, 389-400. https://doi. org/10.1007/s10865-012-9434-0.
Piper, W. E., Joyce, A. S., Azim, H. F., \& Rosie, J. S. (1994). Patient characteristics and success in day treatment. Journal of Nervous and Mental Disease, 182(7), 381-386.

Preacher, K. J., \& Hayes, A. F. (2008). Asymptotic and resampling strategies for assessing and comparing indirect effects in multiple mediator models. Behavior Research Methods, 40(3), 879-891.

Rudden, M., Milrod, B., Target, M., Ackerman, S., \& Graf, E. (2006). Reflective functioning in panic disorder patients: a pilot study. Journal of the American Psychoanalytic Association, 54(4), 13391343.

Schroevers, M., Nyklíček, I., \& Topman, R. (2008). Validatie van de Nederlandse versie van de Mindful Attention Awareness Scale (MAAS) [validation of the Dutch version of the Mindful Attention Awareness Scale (MAAS)]. Gedragstherapie, 41, 225-240.

Shear, J., \& Jevning, R. (1999). Pure consciousness: scientific exploration of meditation techniques. Journal of Consciousness Studies, 6(2-3), 189-209.

Vinnars, B., Barber, J. P., Noren, K., Thormahlen, B., Gallop, R., Lindgren, A., et al. (2007). Who can benefit from time-limited dynamic psychotherapy? A study of psychiatric outpatients with personality disorders. Clinical Psychology \& Psychotherapy, 14(3), 198-210.

Vinnars, B., Thormahlen, B., Gallop, R., Noren, K., \& Barber, J. P. (2009). Do personality problems improve during psychodynamic supportive-expressive psychotherapy? Secondary outcome results from a randomized controlled trial for psychiatric outpatients with personality disorders. Psychotherapy (Chicago, Ill.), 46(3), 362375.

Watson, D., Clark, L. A., \& Carey, G. (1988). Positive and negative affectivity and their relation to anxiety and depressive disorders. Journal of Abnormal Psychology, 97(3), 346-353.

WHOQOL Group. (1998). Development of the World Health Organization WHOQOL-BREF quality of life assessment. Psychological Medicine, 28(3), 551-558.

Publisher's Note Springer Nature remains neutral with regard to jurisdictional claims in published maps and institutional affiliations.

Unfortunately, author Johan Denollet deceased towards the end of the manuscript process. 